\title{
Evidence for paternal DNA transmission to gynogenetic grass carp
}

\author{
Zhuangwen Mao ${ }^{1,2}$, Yeqing Fu' ${ }^{1,2}$, Yude Wang ${ }^{1,2}$, Shi Wang ${ }^{1,2}$, Minghe Zhang ${ }^{1,2}$, Xin Gao ${ }^{1,2}$, Kaikun Luo ${ }^{1,2}$,
} Qinbo Qin ${ }^{1,2}$, Chun Zhang ${ }^{1,2}$, Min Tao ${ }^{1,2}$, Zhanzhou Yao ${ }^{1,2}$ and Shaojun Liu ${ }^{1,2^{*}}$ (D)

\begin{abstract}
Background: Grass carp (Ctenopharyngodon idellus, GC), as the highest-output fish in China, is economically important. The production of gynogenetic grass carp (GGC) will provide important germplasm resource for producing improved GC. At present, knowledge regarding the heterologous sperm DNA in gynogenetic offspring is little. Thus, revealing paternal DNA in GGC at the molecular level would be highly significant for fish genetic breeding.

Result: In this study, ultraviolet-treated sperm of koi carp (Cyprinus carpio haematopterus, KOC, $2 n=100$ ), was used to activate the eggs of GC $(2 n=48)$. Afterwards, cold shock $\left(0-4^{\circ} \mathrm{C}\right)$ was administered for 12 min to double the chromosomes, resulting in GGC. No significant difference ( $p>0.05$ ) was found between GGC and GC in appearance, erythrocytes size and chromosome numbers. However, at the molecular level, a specific microsatellite DNA fragment (MFW1-gynogenetic grass carp, MFW1-G) derived from the paternal parent KOC was found to be transmitted into GGC.
\end{abstract}

Conclusions: For the first time, this study provided an evidence at the molecular level that the DNA fragment derived from the paternal parent occurred in GGC. This finding is of great significance for fish genetic breeding.

Keywords: Ctenopharyngodon idellus, Cyprinus carpio haematopterus, Gynogenesis, Microsatellite DNA, DNA fragment

\section{Background}

Gynogenesis is a type of special reproductive strategy. Artificial gynogenesis usually uses ultraviolet (UV)-treated heterologous sperm to activate the eggs, then the eggs are treated by cold shock or heat shock to double the chromosomes, resulting in gynogenetic progenies.

Yi et al. [1] found microchromosomes from the second-generation gynogenetic gibel carp (Carassius auratus gibelio), in which the gynogenetic gibel carp eggs were activated by UV-treated sperm of blunt-nose black bream (Megaloabrama amblycephala Yin), providing the evidence at the cellular level that the genetic material from heterogenous sperm had the effects on gynogenetic offspring. But this study did not reveal the DNA sequences of the microchromosomes. Peek AS et al. [2] found the paternal genetic material in the gynogenetic albino rainbow trout (Oncorhynchus mykiss)

\footnotetext{
* Correspondence: Isj@hunnu.edu.cn

${ }^{1}$ State Key Laboratory of Developmental Biology of Freshwater Fish, Hunan Normal University, Changsha 410081, Hunan, People's Republic of China ${ }^{2}$ College of Life Sciences, Hunan Normal University, Changsha 410081, Hunan, People's Republic of China
}

activated by the UV-treated sperm of the wild type pigmented brook trout (Salvelinus fontinalis). However, the knowledge regarding the effects of the heterogenous sperm in the gynogenesis is little.

Taxonomically, grass carp (Ctenopharyngodon idellus, GC) belongs to the Cyprinidae, Leuciscinae, while koi carp (Cyprinus carpio haematopterus, KOC) belongs to the Cyprinidae, Cyprininae. GC not only grow quickly but also mainly eat the plant feed [3]. However, the germplasm degradation in GC has led to high disease incidence over the years. In the present study, the newly improved gynogenetic grass carp (GGC) have been formed by cold treatment to double the chromosomes of the GC eggs activated by UV-treated KOC sperm.

Microsatellites DNA, which are also called simple tandem repeats or simple sequence repeats (SSR), consist of a basic sequence containing 10-60 tandem repeats of a repeat unit of 2-6 nucleotides and two conserved flanking regions at the sides of the basic sequence, these features are distributed throughout the genome [4-7]. 
In this study, a pair of primers designated MFW1 (designed by Crooijmans et al. [8]) was used to amplify a specific microsatellite DNA fragment (MFW1-gynogenetic grass carp, MFW1-G), which has been identified in GGC, suggesting that the genetic material derived from the paternal parent might make the effects on the traits of gynogenetic offspring.

\section{Methods}

\section{Samples}

The KOC and GGC were obtained from State Key Laboratory of Developmental Biology of Freshwater Fish, Hunan Normal University, Changsha, Hunan, China; GC was obtained from Wuhu Aquatic Products, Ningxiang, Hunan, China. All the samples used in this study were cultured in ponds and artificially fed. Fish treatments were carried out according to the regulations for protected wildlife and the Administration of Affairs Concerning Animal Experimentation, and approved by the Science and Technology Bureau of China. Approval from the Department of Wildlife Administration was not required for the experiments conducted in this paper. The fish were deeply anesthetized with $100 \mathrm{mg} / \mathrm{L}$ MS-222 (Sigma-Aldrich, St Louis, MO, USA) before dissection.

\section{Gynogenesis}

The female GC reached sexual maturity at 4 years, while the male KOC reached sexual maturity at 2 years. During the reproductive season (from May to June) in 2017 and 2018, 10 mature female GC and 10 mature male KOC were chosen as the maternal and paternal parents, respectively. The gynogenesis experiments were performed according to the methods of Liu et al. [9] and Zhang et al. [10]. First of all, the semen was diluted six times with Hank's balanced salt solution. Then, the diluted semen was spread in culture dishes and placed under UV lamps ZW20S (Cnlight, Guangdong, China) to be sterilized (all processes were conducted in the dark). Next, the UV-treated semen of KOC was used to activate the eggs of mature female GC to develop following the

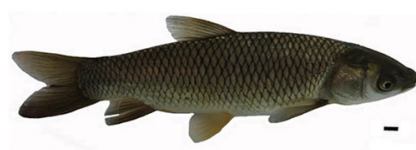

Grass carp $(+)(2 n=48)$

(Ctenopharyngodon idellus)
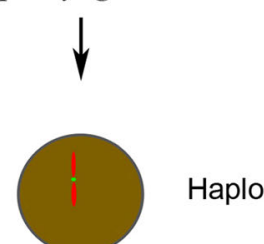

Haploid egg
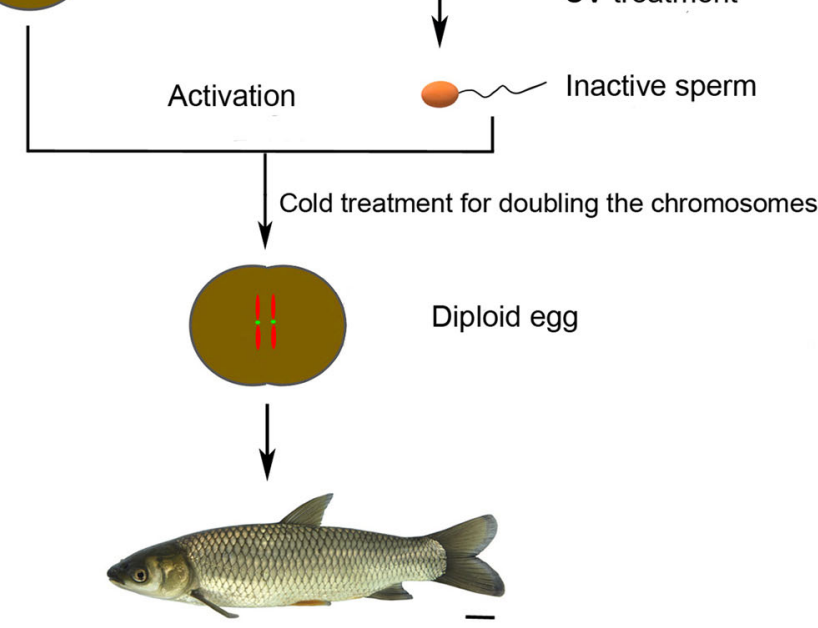

Gynogenetic grass carp $(2 n=48)$
Koi carp $\left(0^{\star}\right)(2 n=100)$

(Cyprinus carpio haematopterus)

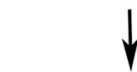

1) Active sperm

UV treatment

ploid egg

Fig. 1 Production processes of GGC. Scale bar, $1 \mathrm{~cm}$ 
A

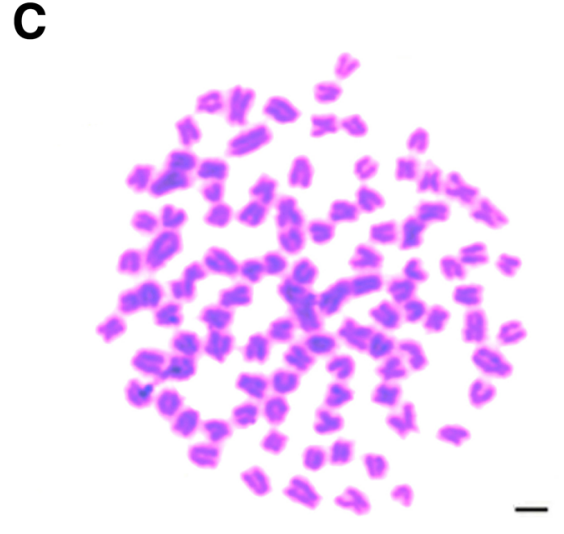

E

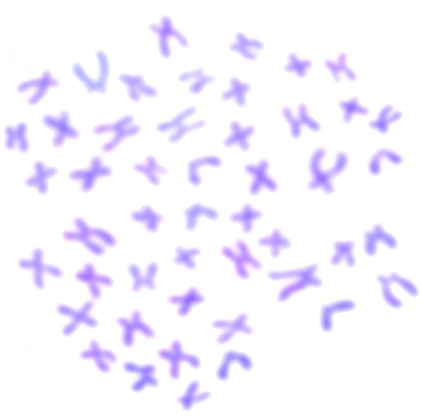

B

I $x \times x \times x \times x \times x$

$\times \times \times \times \times \times \times$

$\operatorname{sm} \pi x \times x \times x \times x \times x$

$x \times x \times \times \times \times x \times$

$\times \times \boldsymbol{\pi}$

sT

D

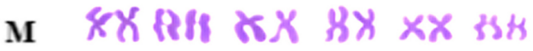

xx $x \times x \times x$

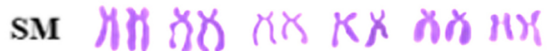

久ช

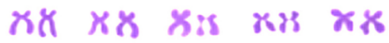

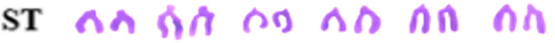

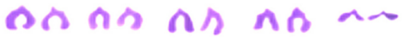

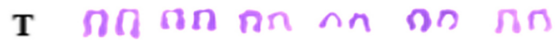

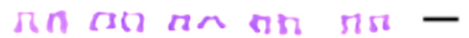

$\mathbf{F}$

M $x x \quad k x \quad k K \quad x x \quad y x$

sM $x x$ xK $k x \quad x x \quad x$

xx $x x$ kx $x x \quad x x$

ถ⿻ $x \times$

st hry na na

Fig. 2 Chromosome spreads at metaphase and corresponding karyotypes of GGC, KOC and GC. a The 48 chromosomes of GGC; $\mathbf{b}$ The karyotype of GGC is $18 m+24 s m+6 s t$; $\mathbf{c}$ The 100 chromosomes of KOC; $\mathbf{d}$ The karyotype of KOC is $22 \mathrm{~m}+34 \mathrm{sm}+22 \mathrm{st}+22 \mathrm{t}$; e The 48 chromosomes of GC; $\mathbf{f}$ The karyotype of GC is $18 m+24 s m+6 s t$. Scale bar, $3 \mu m$

cold shock $\left(0-4{ }^{\circ} \mathrm{C}\right)$ for 12 min to double the chromosomes of haploid eggs. Last, the diploid eggs were incubated in water at $25^{\circ} \mathrm{C}$.

In 2017 and 2018, about 73,000 embryos were selected at random to determine the fertilization rate (number of embryos at the gastrula stage/number of eggs $\times 100 \%$ ) and the hatching rate (number of hatched fry/number of eggs $\times 100 \%)$. The hatched fry were transferred to a pond for further culture.

\section{Measurement of morphological traits}

The traits of 20 GGC, $20 \mathrm{KOC}$ and 20 GC were counted and measured. The countable traits included the numbers

Table 1 Comparison of measurable traits among GGC, KOC and GC

\begin{tabular}{|c|c|c|c|c|c|c|}
\hline Fish type & $\mathrm{BL} / \mathrm{WL}$ & $\mathrm{HL} / \mathrm{BL}$ & $\mathrm{HW} / \mathrm{HL}$ & CPW/CPL & HW/BW & BW/BL \\
\hline GGC & $0.86 \pm 0.02$ & $0.22 \pm 0.02$ & $0.77 \pm 0.04$ & $0.81 \pm 0.07$ & $0.75 \pm 0.01$ & $0.23 \pm 0.01$ \\
\hline KOC & $0.84 \pm 0.04$ & $0.24 \pm 0.01$ & $0.82 \pm 0.06$ & $0.89 \pm 0.04$ & $0.66 \pm 0.06$ & $0.30 \pm 0.03$ \\
\hline GC & $0.84 \pm 0.01$ & $0.22 \pm 0.01$ & $0.70 \pm 0.05$ & $0.79 \pm 0.05$ & $0.73 \pm 0.03$ & $0.21 \pm 0.01$ \\
\hline
\end{tabular}


Table 2 Comparison of countable traits among GGC, KOC and GC

\begin{tabular}{|c|c|c|c|c|c|c|}
\hline Fish type & No. of lateral scales & $\begin{array}{l}\text { No. of upper } \\
\text { lateral scales }\end{array}$ & $\begin{array}{l}\text { No. of lower } \\
\text { lateral scales }\end{array}$ & $\begin{array}{l}\text { No. of abdominal } \\
\text { fins }\end{array}$ & $\begin{array}{l}\text { No. of anal } \\
\text { fins }\end{array}$ & No. of dorsal fins \\
\hline GGC & $41.13 \pm 1.36(39 \sim 42)$ & 6 & 4 & $111+7.38 \pm 0.48(7 \sim 8)$ & $\|1\|+7$ & $\| I+7$ \\
\hline $\mathrm{KOC}$ & $35.67 \pm 1.89(33 \sim 37)$ & 6 & 6 & $\| 1 \mid+8$ & $\| 1 \mid+6$ & $\| I+18.33 \pm 0.94(17 \sim 19)$ \\
\hline GC & $41.5 \pm 0.8(40 \sim 43)$ & 6 & 4 & $1 \|+7.79 \pm 0.41(7 \sim 8)$ & $\|1\|+7$ & $\|1\|+7$ \\
\hline
\end{tabular}

Uppercase Roman numerals indicate the number of the spines, and Arabic numerals reflect the number of soft fins. Numbers before and after the symbol " \pm " represent the mean value and standard deviation of the fin numbers, respectively. " $\sim$ " indicates the measured fin number range

of lateral scales, upper lateral scales, lower lateral scales, dorsal fins, abdominal fins and anal fins. The measurable traits included whole length (WL), body length (BL), body width (BW), head length (HL), head width (HW), caudal peduncle length (CPL) and caudal peduncle width (CPW). In addition, BL/WL, BW/BL, HL/BL, HW/HL, CPW/CPL and $\mathrm{HW} / \mathrm{BW}$ ratios were calculated. The software of SPSS was used to analyze the covariance in the morphological traits in KOC, GC and GGC.

\section{Preparation of chromosome spreads}

To determine the ploidy of GGC, KOC and GC, chromosome counting were performed using kidney tissues from $20 \mathrm{GGC}, 20 \mathrm{KOC}$ and $20 \mathrm{GC}$ at age of 6 months. The chromosomes were prepared in accordance to the method described by Wang et al. [11]. After culturing for two to 3 days at $20-22^{\circ} \mathrm{C}$, the samples were injected one to three times with concanavalin $\mathrm{A}$ at a dose of $6-15 \mu \mathrm{g} / \mathrm{g}$ body weight at an interval of $12-24 \mathrm{~h}$. Two to $3 \mathrm{~h}$ prior to dissection, each sample was injected with colchicine at a dose of 4-6 $\mathrm{\mu g} / \mathrm{g}$ body weight. The kidney tissue was ground in $0.9 \% \mathrm{NaCl}$, subjected to hypotonic treatment with $\mathrm{KCl}(0.075 \mathrm{~mol} / \mathrm{L})$ at $37^{\circ} \mathrm{C}$ for
40-60 min, and then fixed three times in 3:1 methanol-acetic acid. The cells were added dropwise onto cold, wet slides and stained with $4 \%$ Giemsa for 40-60 min. The shape and number of chromosomes were analyzed under a microscope. For each type of fish, 400 metaphase chromosome spreads (20 metaphase spreads from each sample) were analyzed. The preparations were examined under the oil lens at a magnification of $3330 \times$.

Genomic DNA extraction, polymerase chain reaction (PCR) amplification, cloning and sequencing

Total genomic DNA was isolated from the tail fin in 20 GGC, 20 KOC and 20 GC using a DNA extraction kit following the manufacturer's instructions (Sangon, Shanghai, China). A pair of primers (MFW1 F: 5'-GTCC AGACTGTCATCAGGAG-3' and R: 5'-GAGGTGTAC ACTGAGTCACGC-3') were synthesized to amplify the sequence from the mixed genomic DNA extracted from each type of fish. The PCR reactions were carried out in a volume of $30 \mu \mathrm{L}$ with $1 \mu \mathrm{L}$ genomic DNA (approximately $20 \mathrm{ng}$ ), $15 \mu \mathrm{L} 2 \times$ Taq PCR MasterMix (Tiangen, Beijing, China), $13 \mu \mathrm{L} d \mathrm{ddH}_{2} \mathrm{O}$ and $0.5 \mu \mathrm{L}$ of each primer.

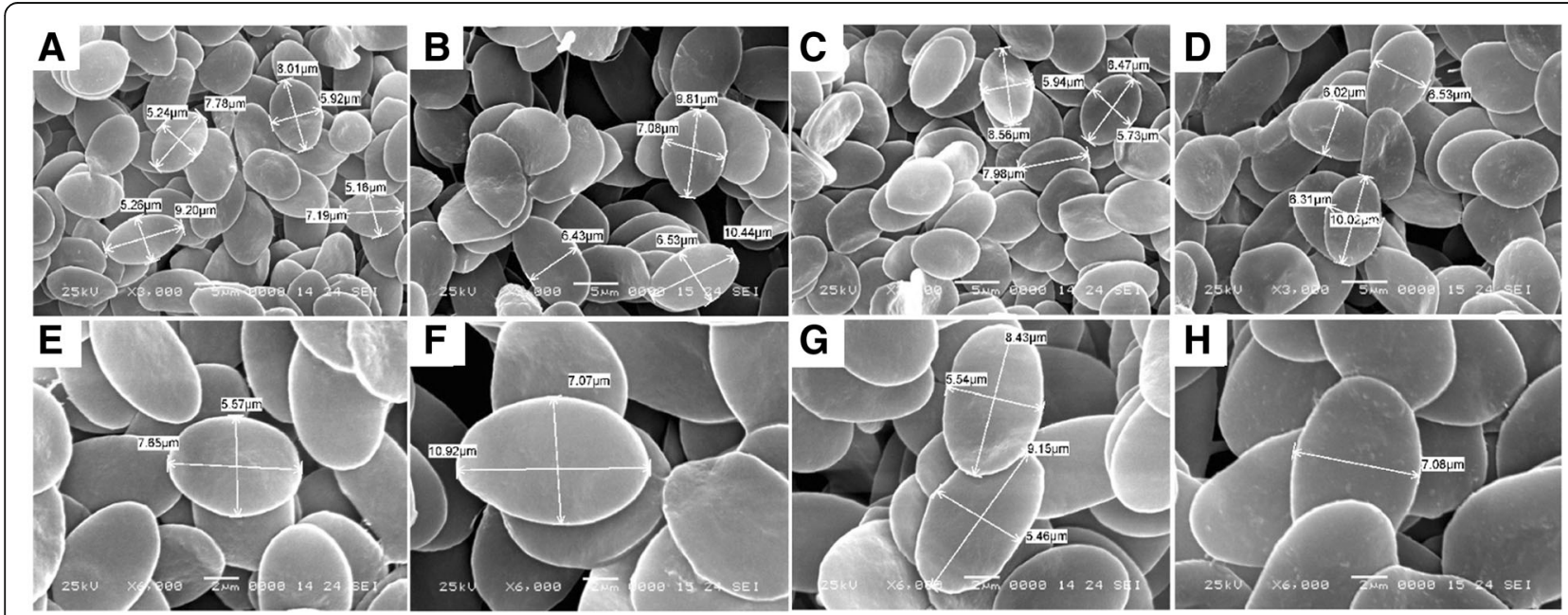

Fig. 3 Erythrocytes of GGC, KOC, GC, and RCC under the SEM. a-d The erythrocytes of GGC, KOC, GC and RCC magnified under 3000 times, respectively. Scale bar in a-d, 5 Hm; e-h The erythrocytes of GGC, KOC, GC and RCC magnified under 6000 times, respectively. Scale bar in e-h, $2 \mu \mathrm{m}$ 
Table 3 Comparison of nuclear volume among GGC, KOC, GC and RCC in erythrocytes

\begin{tabular}{llll}
\hline Fish type & Major semi-axis $(\mu \mathrm{m})$ & Minor semi-axis $(\mu \mathrm{m})$ & Volume $\left(\mu \mathrm{m}^{3}\right)$ \\
\hline GGC & $4.73 \pm 0.48$ & $2.98 \pm 0.22$ & $42.08 \pm 6.75$ \\
KOC & $5.55 \pm 0.25$ & $3.80 \pm 0.32$ & $80.51 \pm 13.73$ \\
GC & $4.33 \pm 0.31$ & $3.09 \pm 0.29$ & $41.64 \pm 8.20$ \\
RCC & $5.37 \pm 0.12$ & $3.87 \pm 0.08$ & $80.43 \pm 1.25$ \\
\hline
\end{tabular}

The temperature profile during amplification was initial denaturation at $94^{\circ} \mathrm{C}$ for $5 \mathrm{~min}$, followed by 35 cycles of $94{ }^{\circ} \mathrm{C}$ for $30 \mathrm{~s}, 53{ }^{\circ} \mathrm{C}$ for $30 \mathrm{~s}$, and $72{ }^{\circ} \mathrm{C}$ for $1 \mathrm{~min}$. A final extension step was performed at $72{ }^{\circ} \mathrm{C}$ for $7 \mathrm{~min}$. The PCR products were separated by polyacrylamide gel electrophoresis (PAGE). The DNA fragments were purified using a gel extraction kit (UNIQ-10 Spin Column DNA Gel Extraction Kit for PAGE, Sangon) and ligated into the pMD18-T vector. The plasmids were transformed into E.coli $D H 5 \alpha$ and purified. The inserted DNA fragments in the pMD18-T vector were sequenced using an automated DNA sequencer (ABI PRISM 3730, Applied Biosystems, Carlsbad, CA). To analyze sequence homology and variation among the fragments amplified from GGC, KOC and GC, the sequences were aligned with BioEdit [12] and Clustal W [13]. The DNA sequences of common carp (GenBank Accession No. LN590690.1) and GC (GenBank Accession No. AY703051.1) were downloaded from the following websites:

(1) The DNA sequences of common carp https://www.ncbi.nlm.nih.gov/nuccore/ LN590690.1? report=fasta

(2) The DNA sequences of GC https://www.ncbi.nlm.nih.gov/nuccore/AY703051.1

\section{Appearance of erythrocytes and measurement of nuclear volume}

The erythrocytes of GGC, KOC, GC and red crucian carp (Carassius auratus red variety, RCC) were dehydrated in alcohol, added dropwise onto slides, desiccated, subjected to atomized gilding and analysed with a JSM-6360LV scanning electron microscope (SEM, JEOL, Japan). The blood smears from each fish were prepared in accordance to the method described by Lu et al. [14]. The erythrocytes from each fish were observed under oil immersion by using an ocular micrometer and the erythrocytes nuclear volume from each fish was calculated by
$(4 / 3) \pi a b^{2}$, where $a$ is the major semi-axis and $b$ is the minor semi-axis of a perfect ellipsoid. The software of SPSS was used to analyze the covariance of the erythrocytes nuclear in KOC, GC and GGC.

\section{Results}

The basic biological characteristics

The UV-treated sperm of KOC was used to activate the eggs of GC. Subsequently, the eggs were treated with cold shock $\left(4^{\circ} \mathrm{C}\right)$ for doubling the chromosomes, which finally developed into GGC (Fig. 1). Forty-eight chromosomes were detected in GGC with a karyotype formula of $18 \mathrm{~m}+24 \mathrm{sm}+6 \mathrm{st}$, which was the same as that of GC (Fig. 2). Countable and measurable traits were compared among GGC, KOC and GC (Tables 1 and 2). GGC retained the characteristics of $\mathrm{GC}$ in the lateral scales, dorsal fins and other countable traits. The body length to whole length ratio of GGC was slightly higher than that of KOC and GC. The head width to head length and tail width to tail length ratios of GGC were between those of KOC and GC. In addition, under the SEM, the erythrocytes of GGC, KOC, GC and RCC were analyzed (Fig. 3). The number of erythrocytes in GGC and GC was greater than that in $\mathrm{KOC}$ and $\mathrm{RCC}$, while the sizes of the erythrocytes in GGC and GC were smaller than that in KOC and RCC. The erythrocytes nuclear volume (Table 3) ratios of KOC to GGC, GC to GGC and KOC to $\mathrm{RCC}$ were $1.91,0.99$ and 1 , respectively. The fertilization rate and the hatching rate in GGC were 25 and $18 \%$ (Table 4 ), respectively.

\section{A DNA fragment from the paternal parent is identified in GGC}

A pair of microsatellite DNA primers designated MFW1 were used to amplify the DNA fragments from GGC and their parents in this study. Five distinct and bright bands were amplified in GGC by the primers of MFW1. In contrast, only four bands were presented in $\mathrm{GC}$ (Fig. 4). After extraction and sequencing, the sequence of MFW1-G, amplified in GGC, was found to be the same $(100 \%)$ as the sequence amplified in the paternal parent KOC and different from that in GC (Fig. 5). The sequence identity of MFW1-G to the corresponding sequence in common carp (Cyprinus carpio, GenBank Accession No. LN590690.1) is 97.5\%. The sequence identity of MFW1-G to that of MFW1 in GC (GenBank Accession No. AY703051.1) is 77.4\%. The results provided the evidence that MFW1-G in GGC was derived from the paternal parent $\mathrm{KOC}$.

Table 4 The fertilization rate and the hatching rate in GGC

\begin{tabular}{lccccc}
\hline Temperature of treatment & No. of eggs & No. of embryos at the gastrula stage & No. of hatching fry & Fertilization rate & Hatching rate \\
\hline $4^{\circ} \mathrm{C}$ & 73,000 & 18,251 & 13,138 & $25 \%$ & $18 \%$ \\
\hline
\end{tabular}




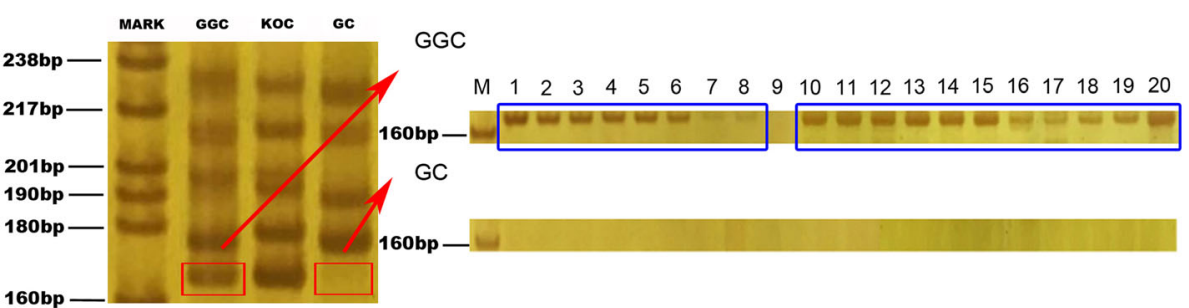

Fig. 4 Amplification result of MFW1 in GGC, KOC and GC. MARK (M): The pBR322 DNA/Mspl Marker; GGC: DNA bands amplified from GGC; KOC: DNA bands amplified from KOC; GC: DNA bands amplified from GC; lanes 1 20: Amplification result of MFW1-G in 20 GGC and 20 GC, respectively (the bands amplified in GGC are in blue boxes and no band has been amplified in GC)

Twenty specimens from GGC and GC respectively were used to repeat the experiments of PCR. According to the results (Fig. 4), MFW1-G was successfully amplified in most of GGC (95\%). In contrast, it was not observed in GC, showing the results of the experiments were high consistent.

\section{Discussion}

Artificial gynogenesis is a useful technology for producing the improved species. With this technology, the living gynogenetic progenies have the potential to form improved characteristics in anti-disease ability and immunity ability with the following possible reasons. First, during the process of gynogenesis, the eggs are activated by UV-treated sperm, suggesting that only high-quality eggs can further develop. Thus, the survival gynogenetic progenies have the potential to form stronger characteristics. Second, during the process of gynogenesis, the eggs activated by UV-treated sperm undergo the cold or heat shock, which heavily damage eggs' development ability, suggesting that the survival gynogenetic progenies have experienced the heavy-stress process. Third, the increased frequencies of some deleterious homologous recessive genes, which makes gynogenetic progenies died. So, the survival gynogenetic progenies have the potential to form improved characteristics. Fourthly, if some paternal genetic materials which insert into the genome or remain in the form of microchromosomes in the gynogenetic progenies, they can result in the "hybrid" effect. In the present study, the gynogenesis hatching rate is very low ( $18 \%$, Table 4$)$, suggesting that the survival progenies of GGC have the potential to get stronger characteristics. On the other hand, the paternal DNA fragment found in GGC suggests the "hybrid" effect, which possibly makes them stronger in some phenotypes.

It is the first time that MFW1-G has been observed in GGC. The sequence of MFW1-G is the same as that of $\mathrm{KOC}$, and also shows very high homology with that of common carp, suggesting that it might be inherited from KOC. One possibility is that some KOC chromosome fragments including the fragment of MFW1-G are integrated or inserted into the genome of GGC. The other possibility is that the chromosome fragments of KOC have developed into microchromosomes separately, which are not observed in the present study. In this study, the MFW1-G has been amplified by PCR using the primers of MFW1 in GGC with a very high frequency (95\%), suggesting that MFW1-G of GGC has been derived from KOC stably. It is possible that the gynogenesis with the DNA fragments derived from the paternal parent in the gynogenetic offspring is a good way to overcome genomic incompatibilities caused by genetic materials from the parents.

\section{Conclusions}

In this study, MFW1-G, a microsatellite DNA fragment, has been observed in GGC, showing the paternal DNA in GGC, which possibly makes GGC

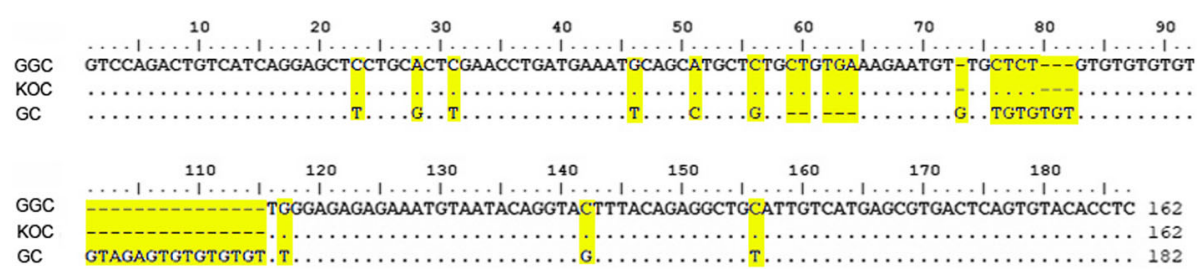

Fig. 5 Nucleotide sequence alignment in GGC, KOC and GC (produced by the same primer MFW1). The nucleotide sequence comparisons in the yellow high light indicate that the nucleotide bases in GGC are the same as those of KOC, but are different from those in GC. The dots indicate sequence identity and the hyphens represent insertions/deletions 
stronger in some phenotypes, providing further evidence that the gynogenesis with the DNA fragments derived from the paternal parent in the gynogenetic offspring will be a possible way to get the improved species. The findings of this study are of great significance for fish genetic breeding.

\section{Abbreviations}

BL: Body length; BW: Body width; CPL: Caudal peduncle length; CPW: Caudal peduncle width; GC: Grass carp; GGC: Gynogenetic grass carp; HL: Head length; HW: Head width; KOC: Koi carp; MFW1-G: MFW1-gynogenetic grass carp; PAGE: Polyacrylamide gel electrophoresis; PCR: Polymerase chain reaction; RCC: Red crucian carp; SEM: Scanning electron microscope; SSR: Simple sequence repeats; UV: Ultraviolet; WL: Whole length

\section{Acknowledgements}

We would like to sincerely thank many researchers who helped to complete this manuscript, including Jinhui Liu, Rurong Zhao, Qingfeng Liu and Liu Cao.

\section{Funding}

This work was supported by the National Natural Science Foundation of China (Grant Nos. 31730098, 31430088), the earmarked fund for China Agriculture Research System (Grant No. CARS-45), Hunan Provincial Natural Science and Technology Major Project (Grant No. 2017NK1031), and the Cooperative Innovation Center of Engineering and New Products for Developmental Biology of Hunan Province (Grant No. 20134486).

\section{Availability of data and materials}

All the datasets used and/or analysed throughout the present study are available from the corresponding author on reasonable request.

\section{Authors' contributions}

SJL designed the whole study and observed the development of the experimental materials. ZWM, YQF, YDW and MHZ performed the experimental work. ZWM, SW, XG, QBQ and ZZY performed the statistical analyses. ZWM, YDW, KKL, CZ and MT collected the experimental materials. ZWM, SW, XG, YDW and ZZY collected the photographs. The manuscript was written and modified by SJL, ZWM, YQF, YDW, SW, QBQ, CZ and MT. All the authors read and approved the final manuscript.

\section{Ethics approval}

All experiments were approved by the Animal Care Committee of Hunan Normal University and followed the guidelines statement of the Administration of Affairs Concerning Animal Experimentation of China. All samples were raised in natural ponds, all dissections were performed under MS-222 anaesthesia, and all efforts were made to minimize suffering.

\section{Consent for publication}

Not applicable.

\section{Competing interests}

The authors declare that they have no competing interests.

\section{Publisher's Note}

Springer Nature remains neutral with regard to jurisdictional claims in published maps and institutional affiliations.

Received: 6 September 2018 Accepted: 26 December 2018 Published online: 07 January 2019

\section{References}

1. Yi MS, Li YQ, Liu JD, Zhou L, Yu QX, Gui JF. Molecular cytogenetic detection of paternal chromosome fragments in allogynogenetic gibel carp, Carassius auratus gibelio Bloch. Chromosom Res. 2003;11(7):665.

2. Peek AS, Wheeler PA, Ostberg CO, Thorgaard GH. A minichromosome carrying a pigmentation gene and brook trout DNA sequences in transgenic rainbow trout. Genome. 1997;40(5):594.
3. Shireman JV, Smith CR: Synopsis of biological data on the grass carp, Ctenopharyngodon idella (Cuvier and Valenciennes, 1844). FAO Fisheries Synopses (FAO) no 1351983

4. Xiao J, Zou TM, Chen L, Liu SJ, Xiao J, Zhang H, Long Y, Yan JP, Zhao RR, Tao M. Microsatellite analysis of different ploidy offspring of artificial gynogenesis in Cyprinus carpio. J Fish Biol. 2011;78(1):150-65.

5. Wei G, Gui JF. Microsatellite marker isolation and cultured strain identification in Carassius auratus gibelio. Aquac Int. 2008;16(6):497-510.

6. Fungtammasan A, Ananda G, Hile SE, Su SW, Sun C, Harris R, Medvedev P, Eckert K, Makova KD. Accurate typing of short tandem repeats from genome-wide sequencing data and its applications. Genome Res. 2015: 25(5):736.

7. Gymrek M. A genomic view of short tandem repeats. Curr Opin Genet Dev. 2017:44:9-16

8. Crooijmans RPMA, Poel JJVD, Groenen MAM, Bierbooms VAF, Komen J. Microsatellite markers in common carp (Cyprinus carpio L.). Anim Genet. 1997;28(2):129-34

9. Liu SJ, Duan W, Tao M, Zhang C, Sun YD, Shen JM, Wang J, Luo KK, Liu Y. Establishment of the diploid gynogenetic hybrid clonal line of red crucian carp XC. carpio. Sci China. 2007:50(2):186-93.

10. Zhang H, Liu S, Zhang C, Tao M, Peng L, You C, Xiao J, Zhou Y, Zhou G, Luo K, et al. Induced Gynogenesis in grass carp (Ctenopharyngodon idellus) using irradiated sperm of Allotetraploid hybrids. Mar Biotechnol. 2011;13(5):1017-26.

11. Wang S, Ye X, Wang Y, Chen Y, Lin B, Yi Z, Mao Z, Hu F, Zhao R, Wang J. A new type of homodiploid fish derived from the interspecific hybridization of female common carp × male blunt snout bream. Sci Rep. 2017:7(1):4189.

12. Hall TA. BioEdit: a user-friendly biological sequence alignment editor and analysis program for Windows95/98/NT. Oxfordshire: Oxford University Press; 1999.

13. Thompson JD, Higgins DG, Gibson TJ. CLUSTAL W: improving the sensitivity of progressive multiple sequence alignment through sequence weighting, position-specific gap penalties and weight matrix choice. Nucleic Acids Res. 1994:22(22):4673-80.

14. Lu WT, Liu SJ, Yu L, Min T, Zhang C, Jing W, Xiao J, Song C, Liu JH, Yun L. Comparative study of erythrocytes of polyploid hybrids from various fish subfamily crossings. Cell Tissue Res. 2009;336(1):159-63.
Ready to submit your research? Choose BMC and benefit from:
- fast, convenient online submission
- thorough peer review by experienced researchers in your field
- rapid publication on acceptance
- support for research data, including large and complex data types
- gold Open Access which fosters wider collaboration and increased citations
- maximum visibility for your research: over $100 \mathrm{M}$ website views per year
At BMC, research is always in progress.
Learn more biomedcentral.com/submissions 